\title{
Indian Hedgehog Regulates Intestinal Stem Cell Fate Through Epithelial-Mesenchymal Interactions During Development
}

\author{
CYNTHIA KOSINSKI, * DANIEL E. STANGE, ${ }^{\star}$ CHUANRUI XU, ${ }^{*}$ ANNIE SY CHAN, ${ }^{\S}$ CORAL HO, * SIU TSAN YUEN, ${ }^{\S}$ \\ RANDY C. MIFFLIN," DON W. POWELL," HANS CLEVERS, ${ }^{\ddagger}$ SUET YI LEUNG, ${ }^{\S}$ and XIN CHEN*
}

*Department of Bioengineering and Therapeutic Sciences, University of California, San Francisco, California; ${ }^{*}$ Hubrecht Institute, KNAW and University Medical Center Utrecht, Utrecht, The Netherlands; §Department of Pathology and Center for Cancer Research, The University of Hong Kong, Queen Mary Hospital, Pokfulam, Hong Kong; and "Department of Internal Medicine, University of Texas Medical Branch, Galveston, Texas

BACKGROUND \& AIMS: Intestinal stem cells (ISCs) are regulated by the mesenchymal environment via physical interaction and diffusible factors. We examined the role of Indian hedgehog (Ihh) in mesenchymal organization and the mechanisms by which perturbations in epithelial-mesenchymal interactions affect ISC fate. METHODS: We generated mice with intestinal epithelial-specific disruption of $I h h$. Gross and microscopic anatomical changes were determined using histologic, immunohistochemical, and in situ hybridization analyses. Molecular mechanisms were elucidated by expression profiling and in vitro analyses. RESULTS: Deletion of intestinal epithelial Ihh disrupted the intestinal mesenchymal architecture, demonstrated by loss of the muscularis mucosae, deterioration of the extracellular matrix, and reductions in numbers of crypt myofibroblasts. Concurrently, the epithelial compartment had increased Wnt signaling, disturbed crypt polarity and architecture, defective enterocyte differentiation, and increased and ectopic proliferation that was accompanied by increased numbers of ISC. Mechanistic studies revealed that $\mathrm{Hh}$ inhibition deregulates bone morphogenetic protein signaling, increases matrix metalloproteinase levels, and disrupts extracellular matrix proteins, fostering a proliferative environment for ISCs and progenitor cells. CONCLUSIONS: Ihh regulates ISC self-renewal and differentiation. Intestinal epithelial Ihh signals to the mesenchymal compartment to regulate formation and proliferation of mesenchymal cells, which in turn affect epithelial proliferation and differentiation. These findings provide a basis for analyses of the role of the muscularis mucosae in ISC regulation.

Keywords: Hedgehog Signaling; ECM; MMP; BMP.

Tn the intestine, epithelial cells undergo repeated pro1 genitor cell proliferation, terminal differentiation, and cell death, a process that requires intestinal epithelial stem cells (ISCs) to engage in a continuous dialogue with neighboring epithelial and mesenchymal cells.1,2 Recent cell lineage tracing experiments have identified Lgr5 and Bmi1 as ISC markers, although they appear to represent 2 distinct ISC populations. ${ }^{3,4}$ The regenerative capacity of
ISCs is directed by structural and biochemical cues received from the ISC microenvironment. ${ }^{5}$ This microenvironment is a complex structure that modulates intestinal homeostasis by maintaining a fine balance between ISC self-renewal and downstream differentiation. While multiple cell types, including endothelial cells, lymphocytes, and muscle cells, may contribute to ISC regulation, the cells generally considered the most important to ISC regulation are intestinal subepithelial myofibroblasts (ISEMFs) because of their close proximity to ISCs. ${ }^{6}$ These mesenchymal cells secrete various factors that favor or restrict ISC self-renewal, including cytokines, matrix proteins, and growth factors, such as bone morphogenetic protein (BMP) antagonists Noggin and Gremlin. ${ }^{7,8}$ Yet how ISEMFs are regulated within the ISC microenvironment, their precise role in fostering ISC self-renewal and proliferation, and whether they are the only major contributors to the mesenchymal ISC microenvironment, remain unclear.

The Hedgehog $(\mathrm{Hh})$ signaling pathway plays a critical role during gut development.9 Expression of the Hh ligands, Sonic Hedgehog and Indian Hedgehog (Ihh), has been detected exclusively in the intestinal epithelium, while expression of Hh target genes, Patched (Ptch1) and Gli1, has been observed in the mesenchyme, including the villus core, muscularis mucosae, and pericryptal myofibroblasts. ${ }^{10,11}$ Both Sonic Hedgehog- and Ihb-null mice display marked gastrointestinal abnormalities, including attenuated smooth muscle layers and intestinal malrotation. ${ }^{10}$ In mice, overexpression of Hedgehog interacting protein (Hhip), a negative regulator of Hh signaling in the gut, leads to mislocalization of ISEMFs and expansion of immature smooth muscle cells. ${ }^{12}$ Furthermore, these mice showed increased cell proliferation and aberrant crypt-like structures, as well as enhanced Wnt activ-

Abbreviations used in this paper: BMP, bone morphogenetic protein; ECM, extracellular matrix; Hh, hedgehog; Hhip, Hedgehog interacting protein; Ihh, Indian Hedgehog; ISC, intestinal stem cell; ISEMF, intestinal subepithelial myofibroblast; MMP, matrix metalloproteinase; SMA, smooth muscle actin.

(1) 2010 by the AGA Institute $0016-5085 / \$ 36.00$ doi:10.1053/j.gastro.2010.06.014 
ity. In converse experiments, enhanced Hh signaling due to conditional deletion of Ptch1 resulted in accrual of colonic myofibroblasts and colonic crypt hypoplasia. ${ }^{13}$

Despite these recent advances, the exact role of Ihh in ISC regulation and gut development remains unclear. Here we find that loss of Ihh signaling causes profound morphological changes to the intestinal mesenchymal compartment, with the most consistent and severe changes occurring near crypt bottoms, where ISCs are located. Our data suggest that the muscularis mucosae might be a novel component of the mesenchymal microenvironment, which acts with myofibroblasts to limit the size of the crypt and ISC pool. Altogether, the results support that Ihh functions as a critical regulator of ISC self-renewal through epithelialmesenchymal interactions.

\section{Materials and Methods (See Supplementary Materials and Methods for Details)}

\section{Mice}

The $I h h^{f l o x} / f l o x$ mice were provided by Dr Beate Lanske of Harvard University. ${ }^{14}$ Villin-Cre mice ${ }^{15}$ and $S m o^{f l o x / f l o x}$ mice $^{16}$ were obtained from the Jackson Laboratory. I $h^{f l o x / f l o x}$ and Villin-Cre mice were mated and the offspring were backcrossed to generate Villin-Cre; Ih $h^{\text {flox/flox }}$ mice. Genotyping was performed by polymerase chain reaction on genomic DNA from tail clips as described previously. ${ }^{14,15}$ The Villin-Cre:Ihbflox/flox pups suffer early lethality and were sacrificed when they displayed lethargy and inability to feed. All mice were housed, fed, and treated in accordance with protocols approved by the committee for animal research at the University of California, San Francisco.

\section{Immunobistochemistry, Immunofluorescence, and In Situ Hybridization}

Animals were euthanized and their intestine was removed and flushed with phosphate-buffered saline. Fixed tissue was embedded in paraffin. Immunostaining was performed using standard protocols after heat-mediated antigen retrieval. Olfm 4 in situ hybridization was performed as described previously. ${ }^{17}$

\section{Statistical Analysis}

Student's $t$ test was used to evaluate statistical significance. Values of $P<.05$ were considered significant.

\section{Results}

\section{Generation of Ihh-Conditional Knockout Mice}

A partial description of Ihh's role in intestinal development has been provided by 2 studies of $\mathrm{Ibh}^{-/-}$ mice: 1 study described a loss of proliferative epithelial cells in $I h b^{-/-}$mice, ${ }^{10}$ while the second study noted an expansion of proliferative epithelial cells in $\mathrm{Ihb}^{-/-}$mice. ${ }^{18}$ However, $I h b^{-1-}$ mice die at birth, precluding any analysis of Ihh's role during postnatal intestinal development. To examine the full role of Ihh in intestinal development, including the critical postnatal period when crypt structures are established, we generated a conditional $I h b$ deficient mouse line by breeding I $h^{f l o x} / f l o x$ mice to Villin-

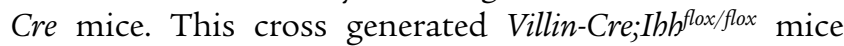
that had intestinal $I h b$ messenger RNA expression levels that were $4 \%$ of control mice, and an absence of Ihh at the protein level (Supplementary Figure $1 A$ and $1 B$ ). The messenger RNA expression level of Ptch1 and Gli1, 2 direct transcriptional targets of $\mathrm{Hh}$ signals, were $19 \%$ and $9 \%$ of the control mice, respectively (Supplementary Figure $1 A$ ), suggesting that $\mathrm{Ihh}$ is the key $\mathrm{Hh}$ molecule mediating $\mathrm{Hh}$ signaling in intestinal tissues, and Sonic Hedgehog or Desert hedgehog cannot replace its function.

Villin-Cre; Ihbflox/flox mice were born alive and appeared normal. However, at P3 it was apparent that the mutant mice were not thriving, as they were noticeably smaller than their control littermates (detailed gross phenotypes are described in Supplementary Figure 1). The majority

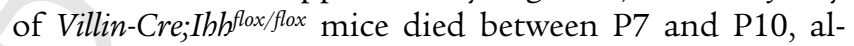
though some managed to survive until P30. The early lethality of Villin-Cre; I $h^{f l o x} / f l o x$ mice is likely caused by malnourishment (Supplementary Figure $1 C$ and $1 D$ ).

\section{Disruption of Intestinal and Colonic Mesenchymal Compartment in Villin-Cre;Ihh ${ }^{\text {flox}}$ flox Mice}

It has previously been reported that Ihh signals in a paracrine direction, moving from its origin in intestinal epithelial cells toward Hh signaling effectors in mesenchymal cells. ${ }^{11}$ We investigated the myofibroblast and smooth muscle changes by $\alpha$-smooth muscle actin $(\alpha$ SMA) immunostaining. The most striking and consistent change observed in the Villin-Cre; Ih flox/flox mice was the loss of a horizontal layer of $\alpha$-SMA-positive cells at the crypt base in the small intestine and colon that corresponds to the muscularis mucosae (Figure $1 A$ and $1 B$; Supplementary Figure 2). The complete loss of muscularis mucosae cells was observed soon after birth and persisted throughout development (Supplementary Figure 2). Because the cellular components of the muscularis mucosae in mice have not been well-characterized, we performed double-labeling experiments for $\alpha$-SMA and desmin, as well as $\alpha$-SMA and vimentin. Myofibroblasts can be distinguished from fibroblasts by their expression of $\alpha$-SMA, and separated from smooth muscle cells by their expression of vimentin and lack of desmin expression. The staining results revealed that in control mice, the cells comprising the thin layer below small intestinal crypts expressed $\alpha$-SMA and vimentin, but not desmin (Figure 1A; Supplementary Figure 2). The results indicate the muscularis mucosae layer absent from the small intestine of Villin-Cre; Ih flox/flox mice is predominantly composed of myofibroblasts. In control colon, $\alpha$-SMA and desmin were coexpressed along the muscularis mucosae 
A
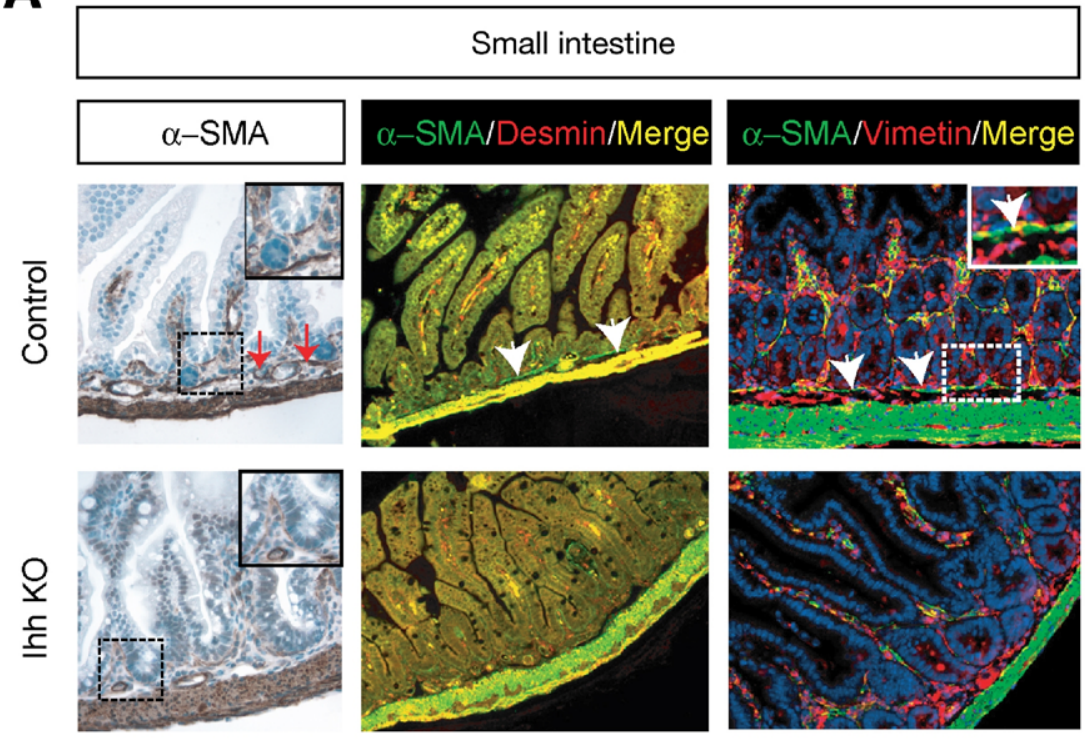

B
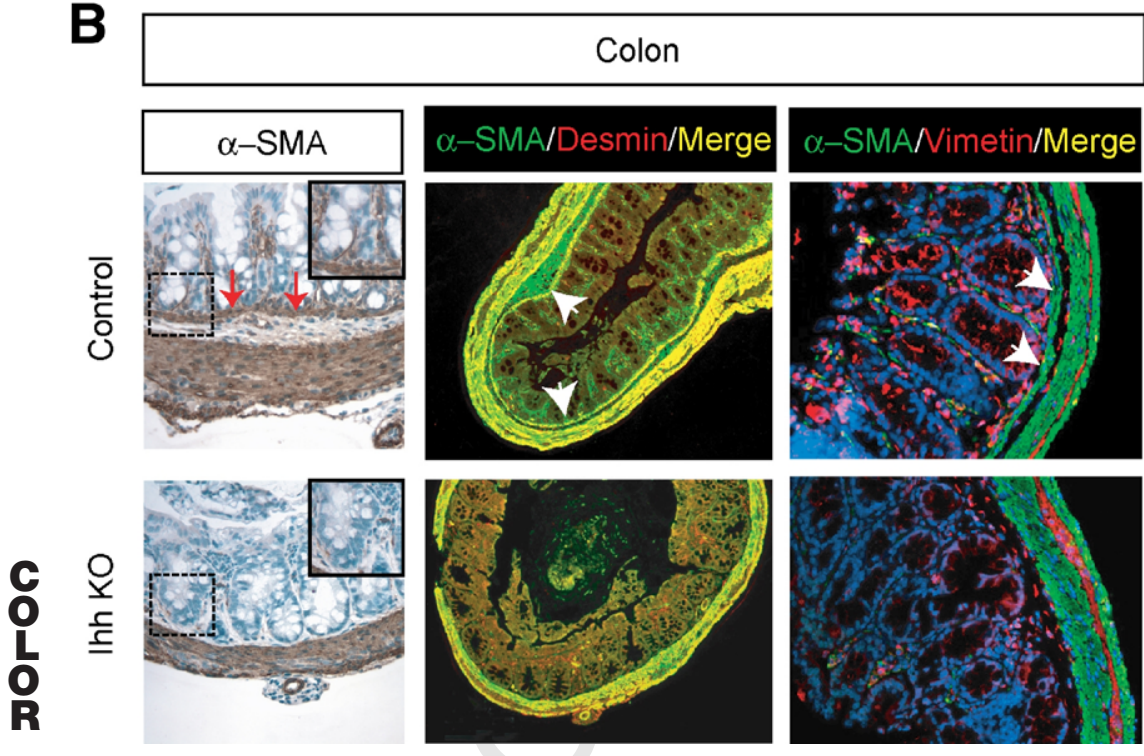

Figure 1. Disrupted mesenchymal compartment in Villin-Cre; Ihh floxflox mice. (A, B) Immunostaining of stromal marker $\alpha$-smooth muscle actin ( $\alpha$-SMA) (left), co-staining of $\alpha$-SMA (green) and desmin (red) (middle) and co-staining of $\alpha$-SMA (green) and vimentin (red) (right) in the small intestine $(A)$ and colon $(B)$, illustrating the loss of muscularis mucosae cells and diminished pericryptal myofibroblasts in Villin-Cre;/Ih floxfflox mice. $\alpha$-SMA staining reveals the muscularis mucosae in control mice (red arrows), whereas in mutant mice no muscularis mucosae is evident. Pericryptal myofibroblasts displayed in the black doted box are shown in detail (inset). The double immunofluorescent stainings show the muscularis mucosae in the small intestine (white arrows) expresses $\alpha$-SMA and vimentin (yellow), revealing that the composition of this layer consists predominantly of intestinal subepithelial myofibroblast (ISEMFs). In the colon, the muscularis mucosae (white arrows) expresses both $\alpha$-SMA and desmin (yellow), indicating that this layer is composed mainly of smooth muscle cells (SMCs). (C) Mesenchymal cell proliferation is observed in P5 control mice (red arrowheads), but is reduced in Villin-Cre;/hhtloxfllox mice; $(D, E)$ The Hedgehog (Hh) inhibitor GANT61 inhibits ISEMF proliferation as assayed by WST-1 $(D)$ and bromodeoxyuridine labeling $(E) .{ }^{\star \star} P<.001$. DMSO, dimethyl sulfoxide; Ihh, Indian Hedgehog; KO, knockout.

underneath colonic crypts (Figure 1B). Noticeably, this cell layer does not express the fibroblast marker vimentin (Figure $1 B$ ), demonstrating the missing muscularis mucosae layer in the colon of Villin-Cre; I hbflox/flox mice is composed of smooth muscle cells (Figure 1B; Supplementary Figure 2).

Because ISEMFs, especially pericryptal myofibroblasts, have traditionally been considered the key mesenchymal cell type that regulates ISCs, we examined the composition of these cells in mutant mice. In the colon, we consistently observed diminished numbers of pericryptal myofibroblasts at the crypt base (Figure $1 B$; Supplementary Figure 2). In the small intestine, loss of pericryptal myofibroblasts was more variable. Additionally, the mislocalization of pericryptal myofibroblasts as described in Villin-Hhip mice ${ }^{12}$ was not observed in Villin-Cre; Ihb flox/flox
114 
mice (Figure 1A; Supplementary Figure 2). Examination of villus core mesenchymal cells revealed a consistent reduction in desmin-positive smooth muscle cells and central core myofibroblasts (Supplementary Figure 2). However, some villi exhibited an expansion of myofibroblasts in regions with superimposed inflammation, suggesting myofibroblast numbers may also be affected by secondary changes. Notably, in P5 control mice, we detected active proliferation of mesenchymal cells surrounding newly forming crypts. In mutant mice, however, mesenchymal cell proliferative activity was profoundly attenuated at this stage (Figure 1C). These data suggest that Ihh loss might impair ISEMF proliferation. To test this hypothesis, we examined in vitro ISEMF cell growth in the presence of GANT61, a small-molecule antagonist of GLI-mediated transcription. ${ }^{19}$ We found GANT61 significantly inhibits ISEMF cell viability and proliferation (Figure $1 D$ and $1 E$ ). Thus, these in vivo and in vitro studies support that Hh signaling is required for ISEMF growth.

Altogether our data indicate that deletion of Ihh results in the disappearance of the muscularis mucosae and fewer ISEMFs surrounding the crypt base, disrupting the key mesenchymal cells that surround ISCs.

\section{Morphological Alterations in the Intestine of Villin-Cre; Ihh ${ }^{\text {flox/flox }}$ Mice}

As we detected marked differences in the mesenchymal compartment surrounding ISCs between control and mutant mice, we sought to determine whether these differences were accompanied by changes in the intestinal epithelium. We found that elimination of Ihh resulted in crypts that appeared wider, loosely organized, and crowded with nuclei (Figure 2A). Villus branching accompanied by aberrant crypt-like structures was also detected in the small intestine of Villin-Cre; Ih $h^{\text {flox/flox }}$ mice (Figure 2A). The ectopic crypt-like structures contained cells that were positive for the proliferation marker Ki67 (Figure 2A). Furthermore, proliferating cells in the mutants greatly outnumbered those in control littermates, with their distribution extending beyond the normal confines of the crypt (Figure $2 A$ ). Crypt fission is a process in which new crypts are produced and is believed to occur in response to stem cell expansion. While crypt fission normally occurs in neonatal mice, we observed a significantly higher incidence of crypt fission in the jejunum of P9 Villin-Cre;Ih flox/flox mice compared to control, revealing major proliferation abnormalities (Figure $2 B$ ). In P30 Villin-Cre;Ihbflox/flox jejunum, we observed marked elongation of crypts with florid proliferation (Figure 2C). Additionally, in some areas, we saw a loss of epithelial maturation, characterized by the absence of villus architecture and proliferative cells reaching the luminal surface (Figure 2C). A similar epithelial phenotype was noted in the colon of Villin-Cre;Ih flox/flox mice in which crypts were dilated with frequent branching and had disturbed orientation and a high degree of proliferation (Supple- mentary Figure 3). Furthermore, in one P30 Villin-Cre; $I h h^{f l o x / f l o x}$ colon, a lesion mimicking a small adenoma with mild dysplasia was detected in the midst of disorganized and disoriented crypts (Figure 2C). These observations suggest that loss of Ihh expression induces florid proliferative events. To determine whether this may eventually culminate in neoplastic transformation would require a study of aged Ihb mutants; however, the early death of Villin-Cre; Ih $h^{f l o x / f l o x}$ mice makes such a study infeasible in our setting.

It is known that a small amount of mosaic Cre expression exists in the colon of Villin-Cre transgenic mice. ${ }^{15}$ In cases in which Villin-Cre;Ihbflox/flox mice survived beyond P15, we noticed normal crypt structures adjacent to dilated crypts in the colon (Figure $2 D$ ). Interestingly, beneath the normal crypt structures $\alpha$-SMA staining detected an intact muscularis mucosae (Figure $2 D$ ), whereas below neighboring dilated crypts, no $\alpha$-SMA staining was detected, revealing an absence of the muscularis mucosae (Figure 2D). Furthermore, Ki67-positive cells were restricted to crypt bottoms in regions where the muscularis mucosae was present (Figure 2D); however, in the absence of the muscularis mucosae, differentiated cells occupied crypt bottoms and proliferative cells were found at crypt bottoms and tops (Figure 2D). These results suggest the muscularis mucosae might influence crypt epithelial fate and polarity, and contribute to ISC regulation.

\section{Loss of Intestinal Epithelial Ibh Signaling Activates Wnt/B-Catenin and Expands the ISC Population}

Given that we observed several manifestations in the Villin-Cre; Ib $h^{f l o x / f l o x}$ mice that were comparable to those seen in mice with increased Wnt signaling, including enhanced epithelial cell proliferation, branched villi, and enlarged crypts, we sought to analyze whether mutant mice displayed increased Wnt activity. ${ }^{20,21}$ Typically, expression of Wnt/ $\beta$-catenin target genes (eg, Cd44, Sox9, EphB2) is restricted to the crypt proliferative compartment (Figure 3A; Supplementary Figure 4). However, in F3 mutant mice Cd44, Sox9, EphB2 expression was highly expressed throughout the crypt and along the villus in the small intestine, as well as along the entire crypt length in the colon (Figure 3A; Supplementary Figure 4). Furthermore, staining for $\beta$-catenin in mutant mice showed increased cytoplasmic staining in crypts and villi, providing additional evidence that the mutants have increased Wnt activity (Supplementary Figure 4).

We next addressed whether the loss of Ihh affected the ISC population by performing in situ hybridization for Olfm4, a marker for small intestinal stem cells. ${ }^{17}$ VillinCre; I $h h^{f l o x / f l o x}$ mice showed an increase in expression of Olfm 4 , as well as an increase in the number of Olfm $4^{+}$cells per crypt compared to control mice (Figure $3 B$ ). A previous study of $I h h^{-/-}$mice suggested that a complete loss of Ihh diminishes the number of ISCs, whereas a second 
A

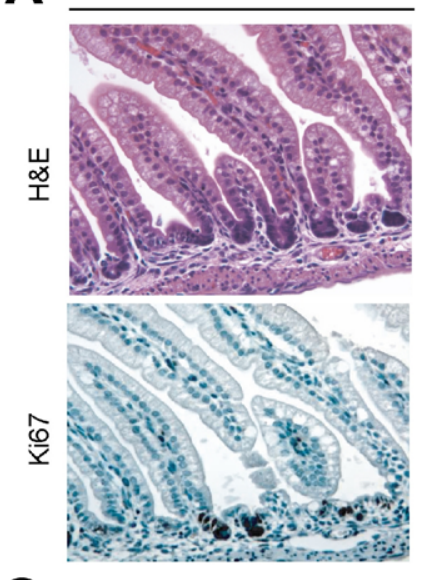

C
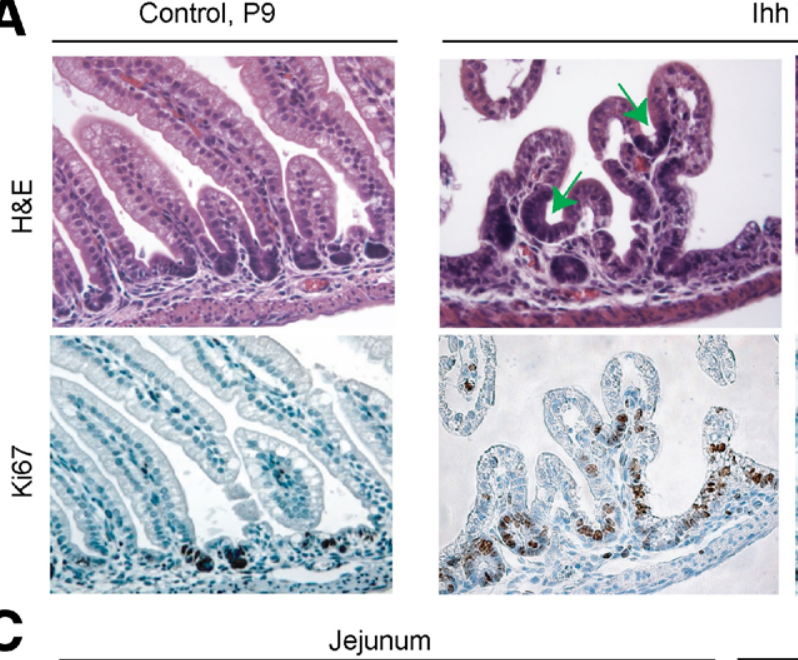

Ihh $\mathrm{KO}, \mathrm{Pg}$

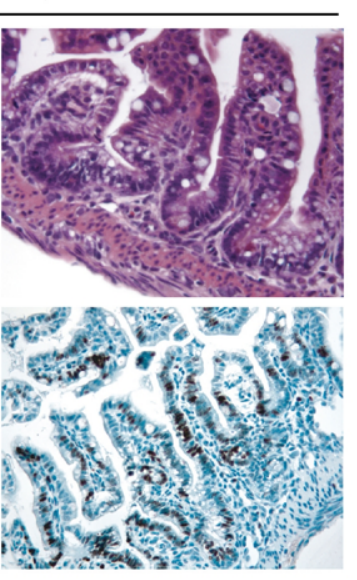

B

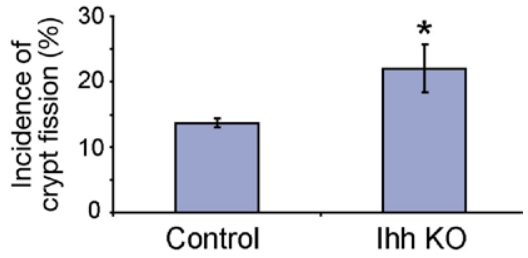

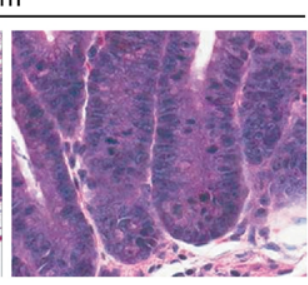

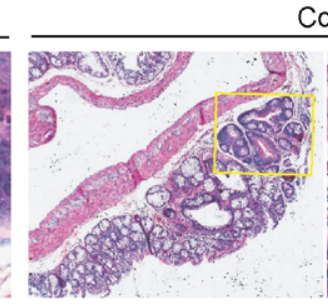

Colon

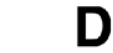

D

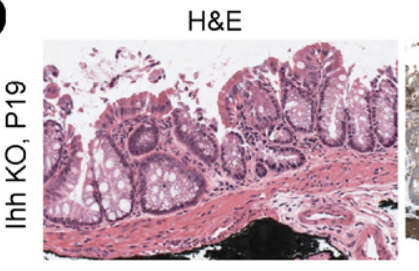

SMA

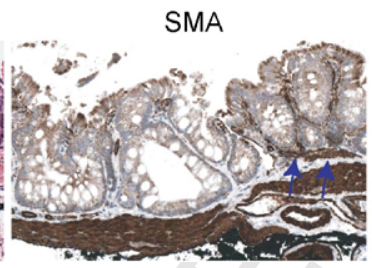

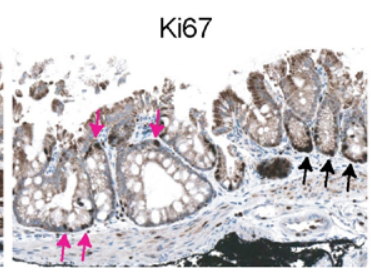

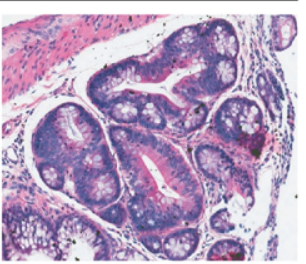

Figure 2. Abnormal intestinal epithelial phenotypes in Villin-Cre;/lhh flox/flox mice. (A) H\&E and Ki67 staining of small intestine tissues from control and Villin-Cre; Ih $h^{\text {floxfllox }}$ mice. Note the sprouting villi and ectopic crypt formation in Villin-Cre; Ihh flox/flox mice (green arrows). (B) Average percentage of crypts in fission in control $(n=3)$ and Villin-Cre; Ihh floxflox mice $(n=3){ }^{\star} P<.05$. (C) H\&E staining shows florid proliferation in the jejunum and adenomatous foci in the colon of P30 Villin-Cre; Ihh floxfllox mice. Yellow boxes designate regions displaying florid proliferation or adenomatous foci. Enlarged images are shown to the right. The jejunum displays focal marked elongation of crypts and a loss of differentiation toward the luminal surface (arrows), whereas adjacent epithelium shows retained maturation at the luminal surface (arrowheads). The colon presents an accumulation of dilated and disoriented crypts that leads to a localized elevated lesion in the mucosa, with evolution into a tubular adenoma with mild dysplasia. (C) Relationship between dilated crypts in colon of Villin-Cre; Ih $h^{\text {flox/flox }}$ mice and loss of muscularis mucosae cells subjacent to crypts revealed through H\&E (left), $\alpha$-smooth muscle actin ( $\alpha$-SMA) (middle) and Ki67 (right) staining. Blue arrows indicate areas with normal crypt morphology above $\alpha$-SMA + muscularis mucosae cells. Typical Ki67 staining pattern is observed in morphologically normal crypts (black arrows), while Ki67 is expressed throughout dilated crypts (pink arrows). Ihh, Indian Hedgehog; KO, knockout.

study suggested the opposite. ${ }^{10,18}$ However, both studies lacked a definitive ISC marker and based their findings solely on a cell proliferation marker. Thus, by utilizing a specific ISC marker, we provide compelling evidence that deactivation of Ihh leads to ISC expansion.

\section{Altered Differentiation of the Absorptive and Secretory Cell Lineages in Villin-Cre; Ihh ${ }^{\text {flox/flox }}$ Mice}

Next, we investigated whether the expansion of epithelial proliferation occurred to the detriment of intestinal epithelial differentiation in Villin-Cre;Ihfflox/flox mice. We examined the differentiation pattern of the 4 different intestinal cell types (ie, enterocytes, goblet, enteroendocrine, and Paneth cells) using specific markers for each cell lineage, as well as electron microscopy (Supplementary Figures 5-7). Our analyses demonstrated that there is an expansion of the secretory cell lineages at the expense of the enterocyte differentiation program in Villin-Cre; Ihh flox/flox mice. Furthermore, we detected an accumulation of vacuolated cells at crypt-villus junctions in the small intestine, indicating the Ihh mutants have some maturation arrest at the enterocyte precursor stage (Supplementary Figure 6).

\section{Inflammatory Response in Villin-Cre;Ihh ${ }^{\text {flox/flox }}$ Mice}

A recent report ${ }^{22}$ showed that chronic Hh inhibition in adult mice results in villus atrophy and profound inflammatory responses in the intestine. We noted patchy 
A

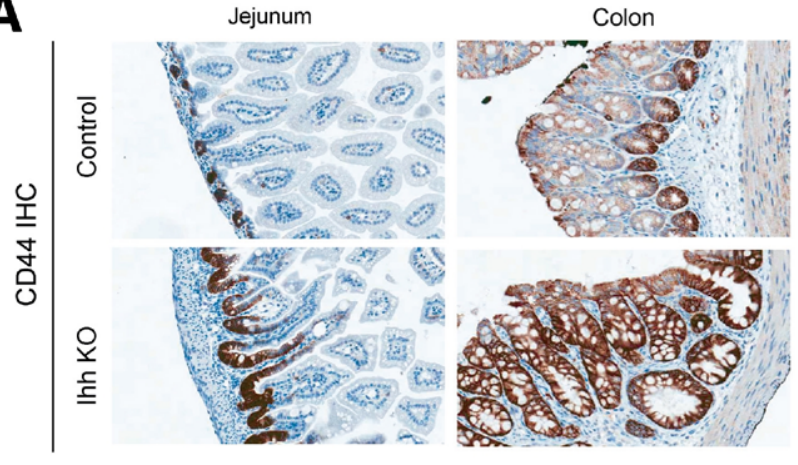

B

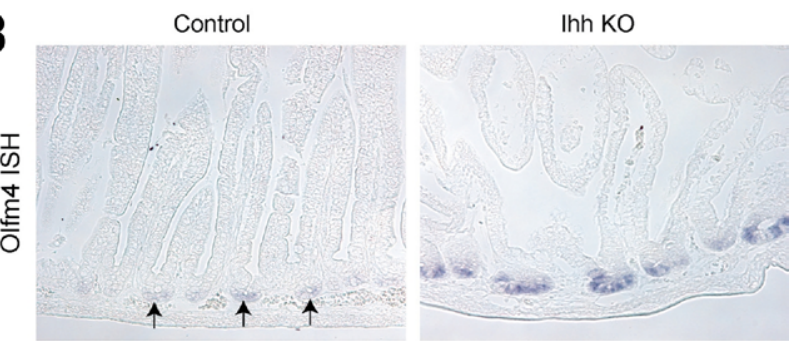

Figure 3. Increased $\mathrm{Wnt} / \beta$-catenin activity and intestinal stem cell expansion in Villin-Cre;/hhtlox/flox mice. (A) Immunostaining of Wnt target gene CD44 in control and Villin-Cre;/hhtloxfllox mice. (B) In situ hybridization (ISH) of the jejunum for Olfm4 in control and mutant mice. Note that the expression pattern of Olfm4 reveals an increase in intestinal stem cell number and expression level in Villin-Cre; Ih $h^{\text {flox/flox }}$ mice. Arrows indicate weak and restricted Olfm4 expression in the control jejunum. IHC, immunohistochemistry; Ihh, Indian Hedgehog; KO, knockout.

and variable inflammatory responses in the small intestine and colon of Villin-Cre; Ih ffloxfflox mice. Early inflammatory changes were observed in regions lined by vacuolated cells at crypt-villus junctions, where breach of epithelial integrity was accompanied by neutrophil infiltration. In acutely inflamed regions, we noticed villus sloughing, resulting in villus loss, mucosal ulceration, dense neutrophil infiltration, and fibrosis (Supplementary Figure 8). Similar surface ulceration with neutrophil infiltration was also noted in the colon (Supplementary Figure 8).

\section{Paracrine Ibh Signaling Responsible for Villin-Cre; Ihh ${ }^{\text {flox/flox }}$ Phenotype}

While some studies suggest Hh signals in an autocrine direction, acting directly on intestinal epithelial cells, ${ }^{18,23}$ more recent reports indicate that Hh signals predominantly in a paracrine fashion in the intestine. ${ }^{11,24}$ To exclude the possibility that the phenotypes we observed in Villin-Cre;Ih flox/flox mice were due to autocrine Hh signaling, we deleted the required hedgehog receptor Smoothened (Smo) in intestinal epithelial cells by crossing Villin-Cre mice with Smoflox/flox mice to generate Villin-Cre; Smoflox/flox mice. We found that Villin-Cre;Smo flox/flox mice were born at normal frequencies and were healthy with no gross abnormalities up to 15 months of age. Microscopic examination revealed normal intestinal and co- lonic architecture (Supplementary Figure 9A). All 4 epithelial cell lineages were well-developed and normal Wnt signaling was observed (Supplementary Figure 9B). The results provide strong genetic evidence that Hh signaling functions strictly in a paracrine manner during gut morphogenesis.

\section{Expression Analysis of Genes Deregulated in Villin-Cre;Ihh ${ }^{\text {flox/flox }}$ Mice}

To investigate the molecular mechanisms underlying the disruption of the mesenchymal compartment and how it leads to abnormal ISC proliferation, we performed expression array analysis of colon samples from control and Villin-Cre; Ihb flox/flox mice. Statistical analysis identified 508 transcripts, including 298 named genes upregulated and 532 transcripts, including 429 named genes down-regulated in Villin-Cre; Ih $h^{f l o x}$ flox mice (Supplementary Table 1). As expected, all Hh signaling targets, such as Gli1, Ptch, and Hhip, were significantly down-regulated in colon samples from mutant mice (Figure $4 A$ ).

Among the genes that were up-regulated in mutants were Wnt targets, including $c-M y c$, Sox-9, and matrix metalloproteinase $(M M P) 7$, as well as the ISC marker $\operatorname{Lgr} 5$ (Figure $4 B$ ). Furthermore, comparison of genes up-regulated in Villin-Cre; Ih $f^{f l o x / f l o x}$ mice with Lgr5 stem cell genes identified several genes that overlap, including $\operatorname{Lgr} 5$, Acot1, Adora1, Sox9, Soat1, and Slc12a2 (Figure 4B). ${ }^{25}$ Genes involved in gut hormones (CCK and glucagon), reflecting changes in enteroendocrine cells, as well as goblet cell marker genes (Spdef, Spink4, Muc2, Gcnt3, and

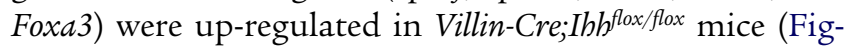
ure $4 C$ ), which is consistent with expansion of secretory cell lineages observed in the mutants. Intriguingly, several MMPs (MMP3, MMP7, MMP8, and MMP10), which are known to degrade extracellular matrix (ECM) proteins and connective tissues, were up-regulated in mutant colon samples (Figure 4D).

Interestingly, the most prominent genes down-regulated in the mutants encode proteins that help support and maintain the intestinal epithelium (Figure $4 D$ and $4 E)$. For example, genes that may provide structural support to ISCs, such as genes involved in smooth muscle development (Myb10, Myb11, myocardin, Mef2c, desmin, etc.) were down-regulated in $I h b$ mutant colons (Figure $4 E$ ). Furthermore, genes encoding ECM proteins (multiple isoforms of collagen and laminin; as well as fibronectin, osteoglycin, versican, nidogen 1, Ecm2, etc.), which provide support, organization, and mechanical signals to the epithelium, were extensively down-regulated in Villin-Cre; I $h h^{f l o x} / f l o x$ mice (Figure $4 D$ ). Additionally, several integrins (Itga1, Itga8, Itga9, Itgav, Itgb6) that attach epithelial cells to the ECM and mediate epithelial cell-matrix interactions were down-regulated in mutant mice (Figure $4 D$ ). Overall, expression analysis demonstrates that the gut mesenchymal compartment is compromised when Ihh is deleted in the intestine, suggesting the possibility that 

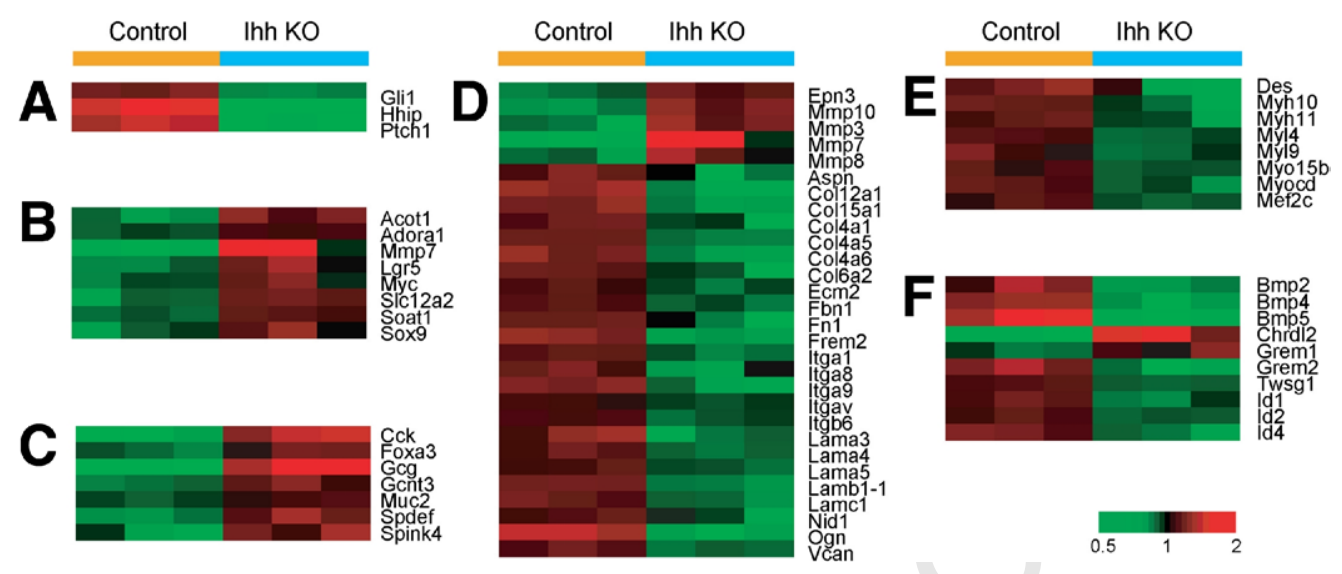

Figure 4. Genes differentially expressed in colon tissues from control and Villin-Cre;/hh flox/flox mice. Heat maps of genes whose expression is significantly increased or decreased in Villin-Cre; Ihh flox/flox mice vs control mice. Each panel represents a functional category: $(A)$ Hedgehog (Hh) target genes; $(B)$ Wnt target and intestinal stem cell-related genes; $(C)$ goblet cell and enteroendocrine-related genes; $(D)$ extracellular matrix-related genes; $(E)$ muscle-related genes; $(F)$ bone morphogenetic protein (BMP) signaling pathway genes. Ihh, Indian Hedgehog; KO, knockout.

disruption of the mesenchymal cells surrounding ISCs may be the key mechanism that leads to abnormal ISC

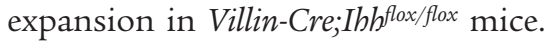

We next analyzed the signaling pathways that have been implicated in gut development, including Notch, BMP, and Ras/MAPK pathway genes. We found that the BMP pathway was one of the major targets for Ihh signaling during gut morphogenesis (Figure $4 F$ ). For example, BMPs, including BMP2, BMP4, and BMP5 were all down-regulated. BMP antagonists showed a more complicated pattern of expression: some were up-regulated, such as Gremlin1 and Chordin-like-2, while others were down-regulated, such as Gremlin2 and Twsg1. Nevertheless, analysis of transcriptional targets of BMP signaling, including $I D 1, I D 2$, and $I D 4$, revealed that all these genes were down-regulated in the mutant mice. Furthermore, immunostaining of phosphorylated Smad1/5 (p-Smad1/5) showed decreased expression of $\mathrm{p}-\mathrm{Smad} 1 / 5$ in the epithelial F5 cells of mutant mice (Figure 5), confirming an overall decrease in BMP signaling in Villin-Cre; Ihf floxffox mice.

\section{Inactivation of Ibb Disrupts Stromal Gene Expression and Basement Membrane Formation}

Given that the gene expression profiling data suggested that the smooth muscle character and ECM components undergo marked changes in Villin-Cre;Ihbflox/flox mice, we decided to investigate how the loss of Hh signaling directly affects expression of muscle and ECMrelated genes in ISEMFs. Quantitative reverse-transcription polymerase chain reaction performed on ISEMFs treated with the Gli transcription inhibitor GANT61 found a significant down-regulation of Mef2c and Myocd, 2 transcription factors known to regulate smooth muscle

F6 development (Figure 6A). These results are consistent with the microarray analysis, suggesting cultured ISEMFs closely resemble the in vivo situation. Furthermore, these findings imply that Hh signals may promote the differentiation of fibroblasts toward a myofibroblast phenotype in the intestine. We next examined the ability of ISEMFs to produce matrix proteins in the absence of $\mathrm{Hh}$ signals. Expression of laminin 4 was significantly downregulated in GANT61-treated ISEMFs (Figure 6A), while other ECM genes, such as fibronectin and collagen, showed no significant change (data not shown). Strikingly, MMPs, including MMP3 and MMP10, were significantly up-regulated in GANT61-treated ISEMFs (Figure 6A). These data suggest that loss of Hh signaling amplifies expression of MMPs in intestinal stromal cells, potentially leading to degradation of ECM components.

These studies indicate that ECM components may be significantly compromised in Villin-Cre; Ih fflox/flox mice. In normal colon, collagen IV expression was detected throughout the lamina propria. In mutant mice, collagen IV staining was significantly reduced in the colon (Figure

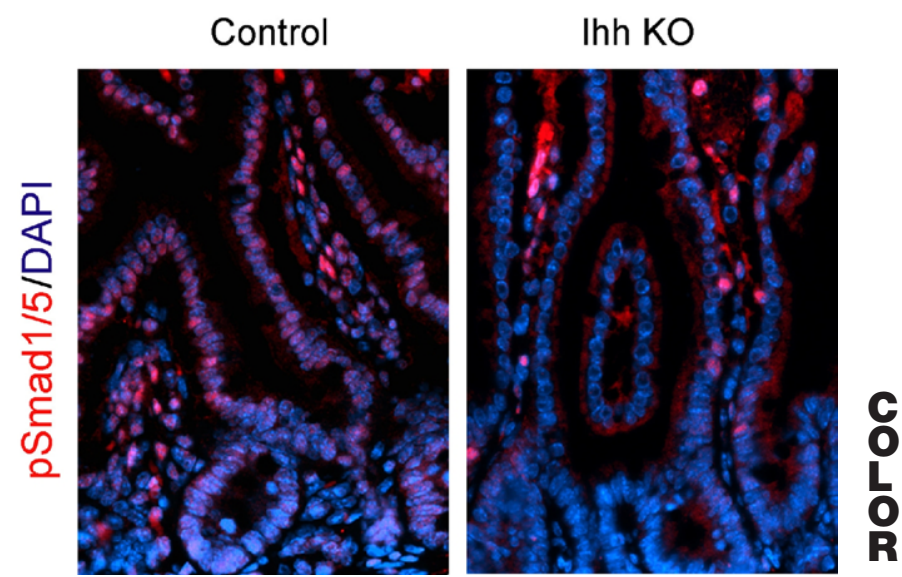

Figure 5. Down-regulation of bone morphogenetic protein (BMP) signaling in Villin-Cre; Ihh floxfflox mice. Immunofluorescent staining of phospho-Smad1/5 in control and Villin-Cre;/hhtlox/flox mice. 
A
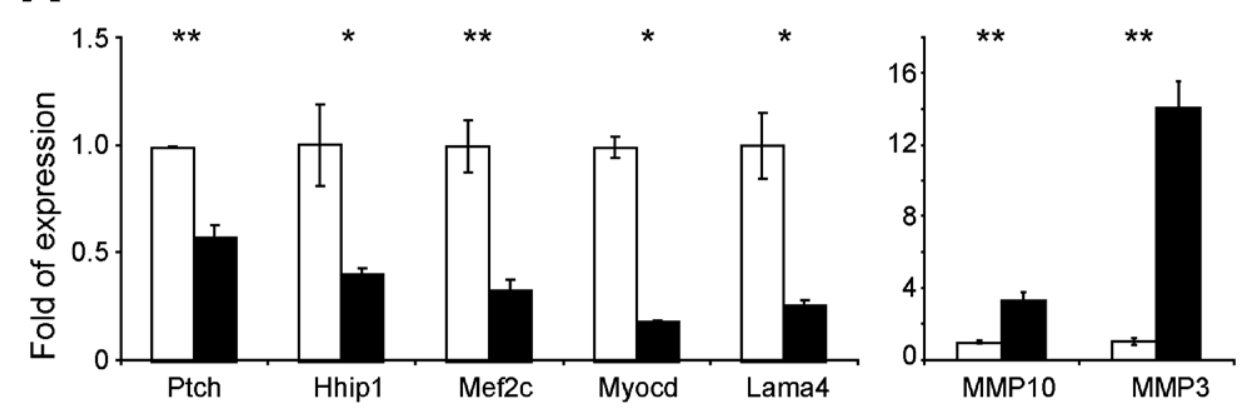

B
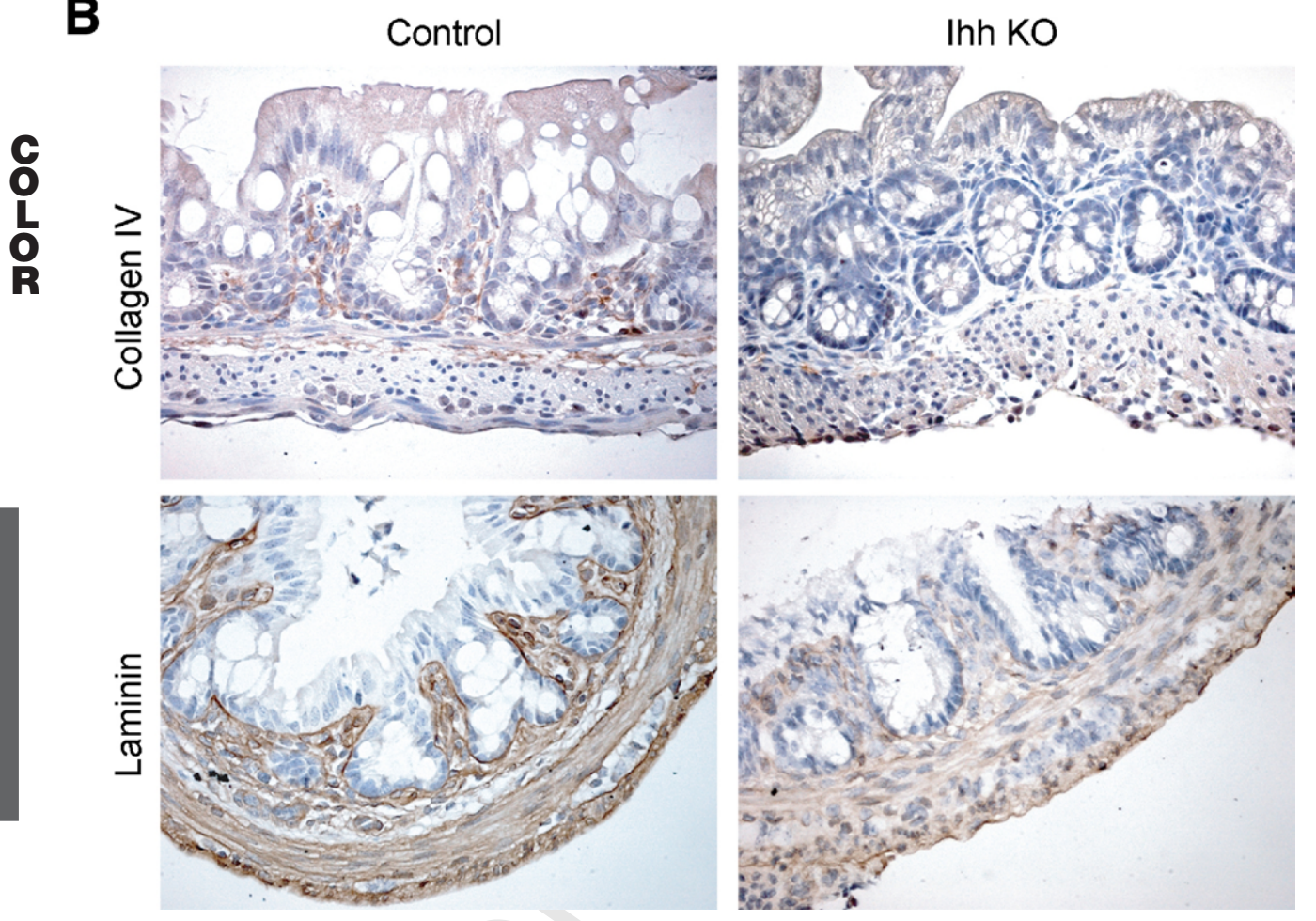

Figure 6. Hedgehog $(\mathrm{Hh})$ regulates stromal gene expression and promotes basement membrane formation. (A) Quantitative reverse-transcription polymerase chain reaction analysis of Ptch1, Hhip, Mef2c, Myocd, Lama4, MMP10, and MMP3 expression in 18Co cells after GANT61 treatment. ${ }^{*} P<.05 ;{ }^{*} P<.01$. (B) Extracellular matrix proteins collagen IV and laminin are expressed in stromal cells and the basement membrane surrounding the crypt in control colon. In Villin-Cre; Ihh floxflox mice, there is decreased expression of both collagen IV and laminin.

$6 B)$. Another basement membrane protein, laminin, was expressed in the lamina propria with intense staining at the epithelial-mesenchymal interface corresponding to the basement membrane in control colon. In contrast, laminin staining appeared diffusely weak and completely absent from the basement membrane in the mutant mice (Figure $6 B$ ). Thus, inactivation of Ihh in the gut leads to down-regulation of ECM genes and matrix protein degradation, resulting in a weakened ECM that is vulnerable to crypt expansion.

\section{Discussion}

The epithelial phenotypes we observed in the Villin-Cre;Ih flox/flox $^{\text {mice }}$ are, overall, consistent with other mouse models that disrupt $\mathrm{Hh}$ signaling during gut morphogenesis, such as mice overexpressing the Hh inhibitor Hhip, or mice with a conditional deletion of Ptch1.12,13 However, none of the previous studies addressed the critical question of whether paracrine $\mathrm{Hh}$ signaling affects ISC self-renewal or whether the epithelial phenotypes are due to the disruption of trans-amplifying/progenitor cells near the crypt base. Our study showed that in Villin-Cre;Ih flox/flox mice, there is a clear expansion of the ISC compartment, providing solid evidence that Ihh regulates ISCs during gut development. Furthermore, our findings indicate that Ihh is the key $\mathrm{Hh}$ ligand mediating the observed epithelial phenotypes in the small intestine and colon after birth. Additionally, our studies with conditional epithelial Smo knockout mice demonstrated that Ihh regulates ISC fates strictly in a paracrine fashion. This statement is corroborated by the fact that there is a profound and consistent disruption of the mesenchymal compartment, especially at the crypt base surrounding ISCs in Villin-Cre;Ih $h^{\text {flox/flox }}$ mice. Altogether, these data suggest that ablation of $I h b$ leads to significant deterioration of the microenvironment surrounding ISCs, which in turn leads to expansion of ISCs and altered epithelial cell differentiation programs. In 
addition, previous studies have suggested that the mesenchymal cells surrounding ISCs are the major source of Wnt and function primarily to maintain ISC proliferation. Here, we propose a different role of the ISC microenvironment, which is to restrain crypt size and prevent abnormal stimulation. Thus, a delicate balance between the proliferative and restrictive activity by mesenchymal cells surrounding ISCs likely exists to refine the shape, size, and function of the gut epithelium to form proper crypt-villus structures.

Pericryptal myofibroblasts are generally considered the key mesenchymal cells that regulate ISCs. On the other hand, cells within the muscularis mucosae layer, despite its vicinity to the ISCs, are not known to be involved in regulating ISCs. In our current study, we find the development of muscularis mucosae cells is strictly dependent on epithelial Ihh signaling, as deletion of Ihh leads to total ablation of the muscularis mucosae in both the colon and small intestine. Our studies suggest that loss of the muscularis mucosae layer may contribute to ISC expansion and deregulation of intestinal epithelial cell differentiation. These findings provide a basis for further investigation of the role of the muscularis mucosae in ISC regulation. For example, in vitro coculture of muscularis mucosae cells with $\mathrm{Lgr}^{+}$ISCs will provide additional evidence of how the muscularis mucosae modulates ISC self-renewal and expansion. In addition, using genomic approaches, one could identify secretory factors produced by the muscularis mucosae. The functions of these secretory factors in regulating ISCs should then be further explored using either knockout mouse models or through in vitro analysis of $\mathrm{Lgr}^{+}$ISCs.

What are the molecular mechanisms behind expansion of the ISC compartment upon Ihh loss during gut morphogenesis? Our genomic analyses indicate that Ihh likely regulates ISC self-renewal and cell fate determination via multiple mechanisms. The reduction of BMP signaling upon Ihh deletion could constitute one possible mechanism that leads to the described phenotype, as BMP signaling normally acts to inhibit ISC self-renewal and repress crypt formation in the gut. ${ }^{7,26}$ Nevertheless, the reduced BMP signaling does not account for all the phenotypes observed in Villin-Cre;Ih flox/flox mice. For example, in villin-noggin mice, in which BMP signaling is completely abrogated, no morphological alternations are detected until 4 weeks of age. ${ }^{26}$ Other factors that likely contribute to the severe phenotypes seen in mutant mice are the complete loss of muscularis mucosae cells and disruption of the ECM that surrounds ISCs. One can imagine muscularis mucosae cells likely provide solid structural support for ISCs at the crypt base. It is also likely that muscularis mucosae cells secrete additional factors that maintain proper ISC number and crypt structure, such as the Wnt antagonist Sfrp2, a down-

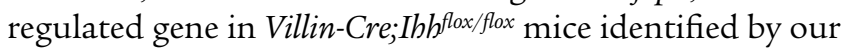

microarray analysis. Additionally, our microarray analysis showed a profound loss of ECM gene expression in Villin-Cre; Ih $h^{\text {flox/flox }}$ mice at the RNA level. The ECM components were further impaired by up-regulation of MMPs, the major enzymes that degrade ECM proteins. Altogether, these mesenchymal compartment changes provide a pro-growth microenvironment for ISCs, promoting ISC expansion and subsequent expansion of the transit-amplifying compartment.

These findings imply a very interesting possibility that Ihh deletion, resulting in the loss of structural ECM integrity, the loss of the muscularis mucosae, and expansion of ISCs may predispose to neoplastic transformation. This is in contrast to many other organs where abnormal activation of the Hh pathway promotes tumor development. ${ }^{27,28}$ Indeed, the role of the $\mathrm{Hh}$ pathway in colorectal cancer remains controversial, with numerous conflicting reports. ${ }^{18,24,29-31}$ Clearly, the precise role of Hh signaling in colorectal tumorigenesis needs to be further clarified.

In a recent study, Zacharias et al reported that chronic Hh inhibition in adult mice resulted in villus atrophy and profound inflammatory responses in the intestine, resembling human celiac disease. ${ }^{22}$ These data suggest that $\mathrm{Hh}$ signaling acts as an important anti-inflammatory factor in the gut. In our study, we also noted the presence of acute inflammation in the intestine of Villin-Cre; Ib flox/flox mice, but mostly in a patchy manner and centered around regions lined by vacuolated cells at the crypt-villus junction in the small intestine and crypt-surface in the colon. We speculate that in the absence of generalized inflammation, the acute inflammatory changes in Villin-Cre;Ihb flox/flox mice most likely results from a weakened mucosal barrier caused by defective enterocyte differentiation. It is possible that the inflammatory changes may also be aggravated by proinflammatory responses released by stromal cells in the absence of Ihh signaling. Our microarray analysis also demonstrated an increased acute inflamma-

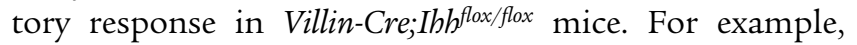
surface markers expressed by neutrophils and/or macrophages, such as CD11 $\beta /$ ITGAM, CD14 and CD177, and proinflammatory cytokines or chemokines, such as interleukin-1 $\beta$, CCL6, CCL9, CCL25 and CXCL5 were all up-regulated in Villin-Cre; Ih flox/flox mice (Supplementary Table 1). Many of these genes overlap with the genes identified by Zacharias et al. However, we detect no resemblance of the inflammatory process in Villin-Cre; $I h^{f l o x / f l o x}$ mice to human chronic inflammatory bowel diseases. Specifically, lymphocytic infiltration, which is characteristically seen in celiac disease or Crohn disease, and significant cryptitis or crypt abscess formation, which is typically seen in ulcerative colitis are all absent in Villin-Cre;Ih flox/flox mice. Altogether, the analysis suggests that Hh signaling may play distinct roles in regulating inflammatory responses during gut development vs homeostasis. 


\section{Supplementary Material}

Note: To access the supplementary material accompanying this article, visit the online version of Gastroenterology at www.gastrojournal.org, and at doi: $10.1053 /$ j.gastro.2010.06.014.

\section{References}

1. Barker N, van de Wetering $M$, Clevers $H$. The intestinal stem cell. Genes Dev 2008;22:1856-1864.

2. van der Flier LG, Clevers H. Stem cells, self-renewal, and differentiation in the intestinal epithelium. Annu Rev Physiol 2009;71: 241-260.

3. Barker N, van Es JH, Kuipers J, et al. Identification of stem cells in small intestine and colon by marker gene Lgr5. Nature 2007; 449:1003-1007.

4. Sangiorgi $E$, Capecchi MR. Bmi1 is expressed in vivo in intestinal stem cells. Nat Genet 2008;40:915-920.

5. Walker MR, Patel KK, Stappenbeck TS. The stem cell niche. J Pathol 2009;217:169-180.

6. Powell DW, Adegboyega PA, Di Mari JF, et al. Epithelial cells and their neighbors I. Role of intestinal myofibroblasts in development, repair, and cancer. Am J Physiol Gastrointest Liver Physiol 2005;289:G2-G7.

7. He XC, Zhang J, Tong WG, et al. BMP signaling inhibits intestinal stem cell self-renewal through suppression of Wnt-beta-catenin signaling. Nat Genet 2004;36:1117-1121.

8. Kosinski C, Li VS, Chan AS, et al. Gene expression patterns of human colon tops and basal crypts and BMP antagonists as intestinal stem cell niche factors. Proc Natl Acad Sci U S A 2007;104:15418-15423

9. van den Brink GR. Hedgehog signaling in development and homeostasis of the gastrointestinal tract. Physiol Rev 2007;87: 1343-1375.

10. Ramalho-Santos M, Melton DA, McMahon AP. Hedgehog signals regulate multiple aspects of gastrointestinal development. Development 2000;127:2763-2772.

11. Kolterud A, Grosse AS, Zacharias WJ, et al. Paracrine Hedgehog signaling in stomach and intestine: new roles for hedgehog in gastrointestinal patterning. Gastroenterology 2009;137:618628.

12. Madison BB, Braunstein K, Kuizon E, et al. Epithelial hedgehog signals pattern the intestinal crypt-villus axis. Development 2005;132:279-289.

13. van Dop WA, Uhmann A, Wijgerde $M$, et al. Depletion of the colonic epithelial precursor cell compartment upon conditional activation of the hedgehog pathway. Gastroenterology 2009; 136:2195-2203 e1-e7.

14. Razzaque MS, Soegiarto DW, Chang D, et al. Conditional deletion of Indian hedgehog from collagen type 2alpha1-expressing cells results in abnormal endochondral bone formation. J Pathol 2005; 207:453-461.

15. Madison BB, Dunbar L, Qiao XT, et al. Cis elements of the villin gene control expression in restricted domains of the vertical (crypt) and horizontal (duodenum, cecum) axes of the intestine. J Biol Chem 2002;277:33275-33283.

16. Long F, Zhang XM, Karp S, et al. Genetic manipulation of hedgehog signaling in the endochondral skeleton reveals a direct role in the regulation of chondrocyte proliferation. Development 2001;128:5099-5108.

17. van der Flier LG, Haegebarth A, Stange DE, et al. OLFM4 is a robust marker for stem cells in human intestine and marks a subset of colorectal cancer cells. Gastroenterology 2009;137: 15-17.
18. van den Brink GR, Bleuming SA, Hardwick JC, et al. Indian Hedgehog is an antagonist of Wnt signaling in colonic epithelial cell differentiation. Nat Genet 2004;36:277-282.

19. Lauth M, Bergstrom A, Shimokawa T, et al. Inhibition of GLImediated transcription and tumor cell growth by small-molecule antagonists. Proc Natl Acad Sci U S A 2007;104:84558460.

20. Wong MH, Rubinfeld B, Gordon Jl. Effects of forced expression of an NH2-terminal truncated beta-Catenin on mouse intestinal epithelial homeostasis. J Cell Biol 1998;141:765-777.

21. van de Wetering $M$, Sancho $E$, Verweij $C$, et al. The beta-catenin/ TCF-4 complex imposes a crypt progenitor phenotype on colorectal cancer cells. Cell 2002;111:241-250.

22. Zacharias WJ, Li X, Madison BB, et al. Hedgehog is an antiinflammatory epithelial signal for the intestinal lamina propria. Gastroenterology 2010;138:2368-2377, 2377.e1-e4.

23. Varnat F, Heggeler BB, Grisel P, et al. PPARbeta/delta regulates paneth cell differentiation via controlling the hedgehog signaling pathway. Gastroenterology 2006;131:538-553.

24. Yauch RL, Gould SE, Scales SJ, et al. A paracrine requirement for hedgehog signalling in cancer. Nature 2008;455:406410.

25. van der Flier LG, van Gijn ME, Hatzis P, et al. Transcription factor achaete scute-like 2 controls intestinal stem cell fate. Cell 2009; 136:903-912.

26. Haramis AP, Begthel $H$, van den Born $M$, et al. De novo crypt formation and juvenile polyposis on BMP inhibition in mouse intestine. Science 2004;303:1684-1686.

27. Jiang J, Hui CC. Hedgehog signaling in development and cancer. Dev Cell 2008;15:801-812.

28. Lauth M, Toftgard R. The Hedgehog pathway as a drug target in cancer therapy. Curr Opin Invest Drugs 2007;8:457-461.

29. Chatel G, Ganeff C, Boussif N, et al. Hedgehog signaling pathway is inactive in colorectal cancer cell lines. Int J Cancer 2007;121: 2622-2627.

30. Berman DM, Karhadkar SS, Maitra A, et al. Widespread requirement for Hedgehog ligand stimulation in growth of digestive tract tumours. Nature 2003;425:846-851.

31. Qualtrough D, Buda A, Gaffield W, et al. Hedgehog signalling in colorectal tumour cells: induction of apoptosis with cyclopamine treatment. Int J Cancer 2004;110:831-837.

Received December 18, 2009. Accepted June 1, 2010.

\section{Reprint requests}

Address requests for reprints to: Xin Chen, XX, Department of Bioengineering and Therapeutic Sciences, University of California, 513 Parnassus Avenue, San Francisco, California 94143. e-mail: xin.chen@ucsf.edu or Suet-Yi Leung, XXX, Department of Pathology, University of Hong Kong, Queen Mary Hospital, Pokfulam, Hong Kong. e-mail: suetyi@hkucc.hku.hk; fax:

\section{Acknowledgments}

We thank Dr Beate Lanske of Harvard University for providing us with the $I h h^{\text {flox/flox }}$ mice and Sandra Huling of the UCSF Liver Center for histology and electron microscopy support.

Author contributions: Study concept and design: CK, SYL, XC. Acquisition of data: CK, DES, CX, ASC, CH. Analysis and interpretation of data: CK, DES, STY, RCM, PWD, HC, SYL, XC. Drafting of the manuscript: CK, DES, SYL, XC. Provide reagents: RCM, PWD, HC. Study supervision: XC, SYL.

Transcript profiling: The raw data of microarray experiment are available at Gene Expression Omnibus (GEO, http://www.ncbi. nIm.nih.gov/projects/geo) under the accession number of GSE18393. 
562 AQ:6 Conflicts of interest

563

564

565

566 the Hong Kong Special Administrative Region (Project No. HKU7524/ 06M) to S.Y.L. and X.C.; a grant from Strategic Research Theme on Cancer from The University of Hong Kong to S.Y.L. C.K. is supported by a stem cell fellowship provided by the California Institute for Regenerative Medicine; and D.E.S. is supported by Deutsche Forschungsgemeinschaft (DFG, Sta1065/1-1). The funding agencies have no role in data collection, analysis, and interpretation.
This work is supported in part by National Institutes of Health grants R21DK069309 and R01CA136606 to X.C.; R01CA127229 and R01DK55783 to D.W.P.; a grant from the Research Grants Council of 